\title{
Review of Upscaling Methods for Describing Unsaturated Flow
}

\author{
Brian D. Wood
}

September 2000

\section{RECEIVED \\ OCT 02000 \\ OS TI}

\section{Prepared for}

the U.S. Department of Energy

under Contract DE-AC06-76RLO 1830

Pacific Northwest National Laboratory

Richland, Washington 99352 


\section{DISCLAIMER}

This report was prepared as an account of work sponsored by an agency of the United States Government. Neither the United States Government nor any agency thereof, nor any of their employees, make any warranty, express or implied, or assumes any legal liability or responsibility for the accuracy, completeness, or usefulness of any information, apparatus, product, or process disclosed, or represents that its use would not infringe privately owned rights. Reference herein to any specific commercial product, process, or service by trade name, trademark, manufacturer, or otherwise does not necessarily constitute or imply its endorsement, recommendation, or favoring by the United States Government or any agency thereof. The views and opinions of authors expressed herein do not necessarily state or reflect those of the United States Government or any agency thereof. 


\section{DISCLAIMER}

Portions of this document may be illegible in electronic image products. Images are produced from the best available original document. 


\section{Summary}

The representation of small-scale features can be a challenge when attempting to model unsaturated flow in large domains. Upscaling methods offer the possibility of reducing the amount of resolution required to adequately simulate such a problem. In this report, the various upscaling techniques that are discussed in the literature are reviewed. The following upscaling methods have been identified from the literature: 1) stochastic methods, 2) renormalization methods, and 3) volume averaging and homogenization methods; in addition, a final technique, full resolution numerical modeling, is also discussed. Each of these techniques has its advantages and disadvantages. The trade-off is a reduction in accuracy in favor of a method that is easier to employ. For practical applications, the most reasonable approach appears to be one in which any of the upscaling methods identified above may be suitable for upscaling in regions where the variations in the parameter fields are small. For regions where the subsurface structure is more complex, only the homogenization and volume averaging methods are probably suitable. With the continual increases in computational capacity, full-resolution numerical modeling may in many instances provide a tractable means of solving the flow problem in unsaturated systems. 


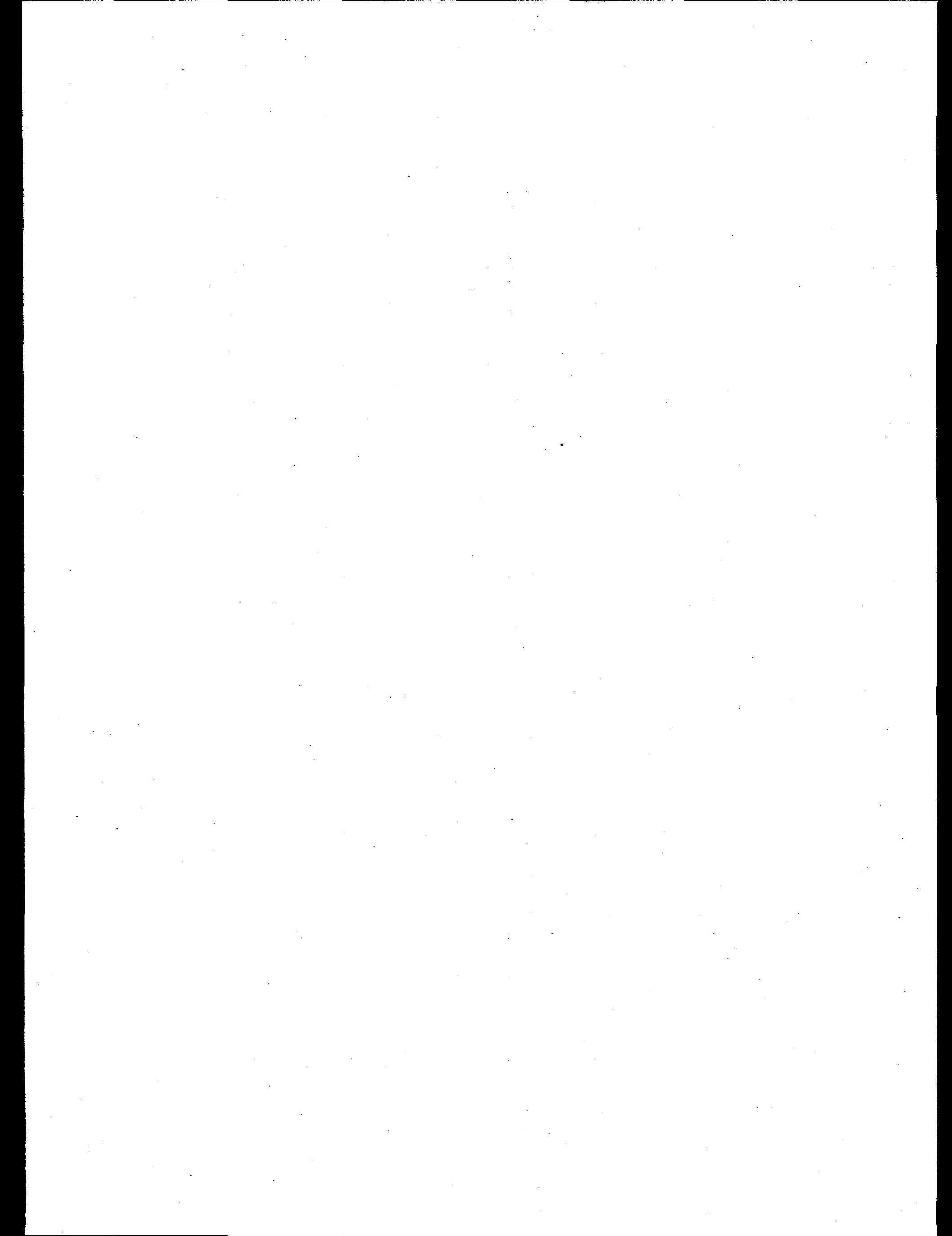




\section{Contents}

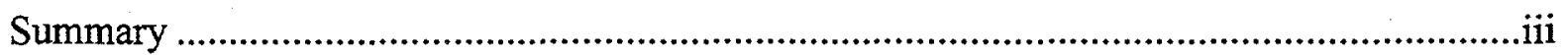

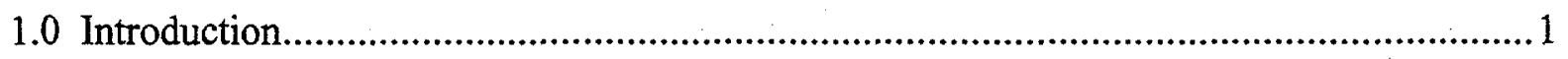

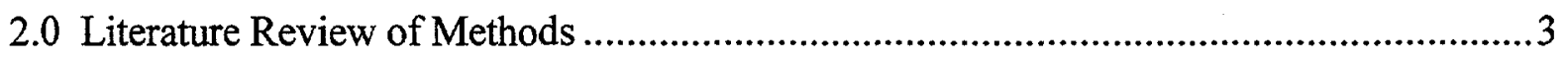

2.1 Stochastic Techniques .......................................................................................4

2.2 Renormalization Techniques .....................................................................4

2.3 Volume Averaging and Homogenization Techniques ..........................................6

2.4 Full-Resolution Numerical Modeling .......................................................... 8

3.0 Method Recommendations ........................................................................... 11

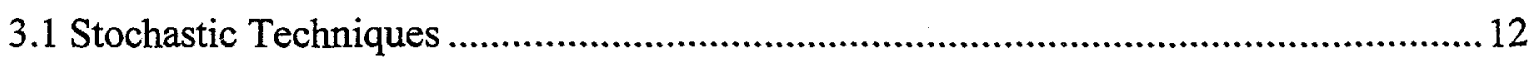

3.2 Renormalization Techniques .................................................................... 12

3.3 Volume Averaging and Homogenization Techniques ...................................... 13

3.4 Full-Resolution Numerical Modeling ........................................................ 13

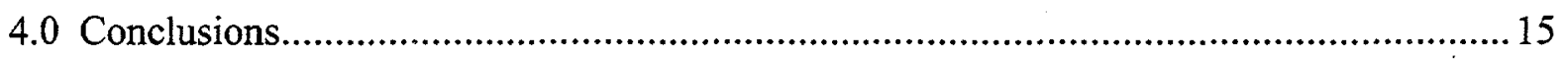

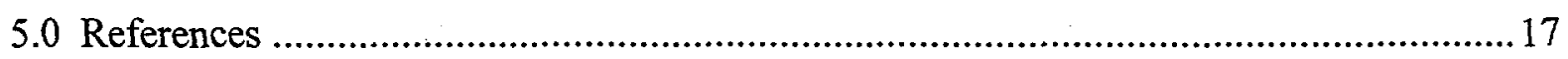





\subsection{Introduction}

The stochastic description of flow and transport in the subsurface is now a mature area of research in hydrology, and the subject is represented by an extensive literature. Remarkably, many of the established methods pertain primarily to a subset of possible subsurface structures, specifically those that can be represented by normal or lognormal parameter distributions. Also remarkable is the fact that, although many mathematically rigorous methods have been developed, only a relatively small subset of these has been adopted for use in practice.

One of the needs for the immobilized low-activity waste (ILAW) project is the ability to predict the performance of containment trenches over long periods of time. Numerical simulations of fluid flow and dissolved species transport are necessary for these performance assessments. Because the construction of waste isolation trenches may contain several disparate characteristic length scales (e.g., the thickness of the materials between waste containers may be on the order of centimeters, whereas the trench may be hundreds of meters long), the computational costs for conducting high-resolution numerical simulations may be prohibitive. The purpose of this report is to summarize some of the upscaling methods that may be suitable for use in ILAW design simulations. Upscaling methods can allow accurate simulations to be conducted with reduced computational costs by reducing the resolution required. This is done by replacing the fine-resolution description of the problem by a more coarsely resolved one; in these methods, the coarse grid properties have been determined from the fine grid properties through a formal upscaling procedure.

This report is organized as follows. In Section 2, the literature is reviewed, and various methods for upscaling flow in the unsaturated zone are identified, summarized, and compared. In Section 3, recommendations for particular methods most suitable for ILAW design purposes are made. Finally, in Section 4, a brief summary is provided. Although the transport of both fluids and dissolved solutes are pertinent to these design calculations, for this report only the upscaling of fluid flow is considered; the upscaling of solute transport will be considered in a future report.

The development of upscaled flow parameters and upscaled solute transport parameters may require very different perspectives. The importance of this point is that upscaled representations for the flow problem will provide the correct coarse-grid flow field only; such a flow field will not provide useful information for predictions of solute transport. 



\subsection{Literature Review of Methods}

The literature describing upscaling flow in heterogeneous media is enormous, and the variety of methods appears to be substantial. In fact, there are only a handful of methods that are actually used in these developments. In the broadest terms, the methods that are used can be grouped into the following categories: 1) stochastic (regular perturbation) methods, 2) renormalization theory, and 3) volume averaging/homogenization methods. In this section, the literature for each of these methods will be reviewed briefly, and additional commentary for the methods will be added when appropriate. In addition to the previously mentioned categories, a non-upscaling method will also be discussed. This method will be referred to as full-resolution numerical modeling. In the discussion that follows, two definitions for upscaled properties will be used (following Renard and de Marsily 1997): equivalent properties and effective properties. Although this division is somewhat tenuous, it is useful for our discussion.

The term equivalent properties is usually used to indicate a property that has been averaged over a subdomain (often referred to as a block), where some constraints have been imposed for calculating the equivalent property. For the example of saturated conductivity, the constraints are that the mean boundary fluxes must be the same and the energy dissipated is equivalent (Renard and de Marsily 1997). For equivalent parameters, no statistical constraints are imposed upon the medium, so the equivalent properties of the medium may depend on the particular conditions that are imposed on the block boundaries. Equivalent parameters are generally sought when upscaling a fine-grid parameter field for calculating a numerical solution on a coarser grid.

The term effective properties is generally reserved for media in which certain statistical constraints have been imposed. The most common constraints are spatial stationarity and ergodicity in the mean and covariance structure. Generally, such constraints are imposed so that one can obtain the asymptotic behavior of the medium. This means only that the statistics are sufficiently sampled by the method that the effective parameter being determined is a function of only the parameter field statistics (and not of the particular physical distribution that would be implied by any particular realization of these statistics). Under these conditions, information from the boundaries is either moot (i.e., the field is considered to be of infinite or semi-infinite extent) or boundary information is lost so rapidly as one moves toward the interior of the domain that the particular boundary conditions that are imposed do not affect the calculation of the effective parameter. Effective parameters are useful because they are functions of only the statistics of the field, rather than the particular details of any realization of a field.

For some applications, the division between equivalent and effective becomes meaningless because a combination of volume averaging and statistical averaging techniques is used (e.g., Kitanidis 1990). Therefore, in the discussions that follow, these two terms will be used when appropriate but with the caveat that the meaning may not always be precise. 


\subsection{Stochastic Techniques}

Stochastic techniques are probably the most familiar methods for upscaling to hydrologists. This method has been used extensively for determining the effective saturated conductivity of heterogeneous porous media (e.g., Cushman 1990; Dagan 1989; Dagan and Neuman 1997; Gelhar 1993); results for the effective saturated conductivity are summarized in detail in these references. For unsaturated flow, the approach used to develop the effective conductivity usually assumes that the conductivity-pressure relationship takes the form

$$
K(\psi, \mathbf{x})=K_{s}(\mathbf{x}) \exp (-\alpha(\mathbf{x}) \psi)
$$

where $K(\psi, \mathbf{x})$ is the hydraulic conductivity, $K_{s}(\mathbf{x})$ is the saturated hydraulic conductivity, and $\alpha(\mathbf{x})$ is the slope of the unsaturated conductivity function. This functional form is chosen primarily for tractability by analytical techniques rather than its applicability to the field. In these analyses, the parameters $K_{s}(\mathbf{x})$ and $\alpha(\mathbf{x})$ are treated as random fields with lognormal statistics. This form for the conductivity-pressure relationship is combined with a first-order perturbation analysis to yield estimates of the effective conductivity.

The first efforts for a stochastic perturbation analysis of unsaturated flow were developed by Yeh et al. (1985a, 1985b, 1985c) for steady flow conditions with anisotropic parameter field statistics. The method involved a conventional linear perturbation analysis that was solved using spectral representation techniques. Later, Mantoglou and Gelhar (1987a, 1987b, 1987c) extended this approach to transient flows and developed representations for the upscaled soil moisture content, soil moisture capacity, and conductivity. This analysis included an explicit representation for the possibility of hysteresis induced by heterogeneity. Pollmann et al. (1991) tested this theory through numerical experiments, and they agreed qualitatively with predictions from the theory. They were able to carefully identify the limitations associated with the theory developed by Mantoglou and Gelhar (1987a, 1987b, 1987c). Russo (1992) was able to combine the theory of Yeh et al. (1985a, 1985b, 1985c) with the concept of the equivalent block permeability to explore how large a heterogeneous domain must be so that the equivalent hydraulic conductivity converged to the effective hydraulic conductivity. In this work, it was determined that the diagonal components of the equivalent conductivity tensor converged with those for the effective conductivity tensor when more then 20 integral scales were included. Finally, Mantoglou (1992) was able to extend his previous analysis of unsaturated flow to conditions of nonstationary statistics. This theory was tested by applying it to field observations (Jensen and Mantoglou 1992); the stochastic model was able to predict the average system behavior reasonably well.

\subsection{Renormalization Techniques}

Renormalization techniques have become a popular method of determining the equivalent permeability because they can be very easy and computationally inexpensive to implement. There are in fact two distinct approaches that are called "renormalization" techniques. The first technique (which will be referred to in the remainder of the discussion as "renormalization-field theory") uses elements of field theory to develop series solutions for the effective parameter of 
interest (usually the hydraulic conductivity). In essence, this technique can be viewed as an improved version of the linear perturbation expansion technique; however, it also suffers from many of the same limitations as the perturbation expansion technique. The second technique (which in the remainder of the discussion will be referred to as "standard renormalization") can be viewed as a solution obtained via a finite difference scheme. Each of these techniques is described in more detail below.

In the renormalization-field theory techniques, the methods of field theory (originally developed for applications to quantum electrodynamics) are used for development of a perturbation expansion. In essence, the method consists of developing a Green's function solution to the governing differential equation (e.g., the flow equation), and then splitting this solution up into a random part and a constant part. The solution is iterated, yielding a series expansion for the Green's function. After some algebraic manipulation, the (averaged) Green's function is related to the effective conductivity, and the problem is closed by finding suitable approximations for the Green's function expansion. It should be noted that this approach is generally only useful for determining the effective hydraulic conductivity, and the approach requires that one adopt a field with a small variance in the parameter of interest (i.e., the socalled small perturbation assumption). To date this technique has been applied only to the estimation of effective saturated hydraulic conductivities, but there is no reason to expect that it would not also be suitable for the development of the effective hydraulic conductivity function for unsaturated conditions, since it has been shown that regular perturbation techniques will work.

Despite its seemingly complex mathematical approach, the renormalization-field theory technique has been explored by many researchers. The first application of this technique was by King (1987), who approached the problem using the tools and notation borrowed directly from theoretical physics. A more recent application developed by Christakos et al. (1995), who used Feynman diagrams for expansion of the perturbation series, puts the problem in a more strictly hydrologic context. This method has been reformulated somewhat by an approach that uses an approximation to the series representing the Green's function (Hristopulos and Christakos 1997), thus making evaluation of the solution easier.

In the standard renormalization technique, a sequential process of upscaling is used to translate fine-grid information up to coarse grids. At each level of upscaling, grid blocks from the previously defined level are grouped, and upscaled parameter properties are determined using finite difference-like techniques. At each step in the upscaling, particular conditions must be imposed at the boundaries of large-scale blocks; it is not always clear what the proper boundary conditions should be for the upscaling process, and this poses one of the primary difficulties in this method. However, because this method is generally simple to implement (with analytical methods available for the successive upscaling in many cases), it has been implemented frequently.

The standard renormalization technique also has its roots in theoretical physics, but the first application to subsurface hydrology is generally attributed to King (1989), who used the method for calculating the equivalent permeability for saturated flow. Explicit calculations of the error terms that might be involved in this technique have been explored by King and Williams (1994). 
Gautier and Naetinger (1994) have proposed a method for developing the equivalent conductivity tensor by imposing periodic boundary conditions. A related method based on a percolation theory applied to random resistor networks has been developed by Hunt (1998). In this approach, the effective conductivity is determined on the basis of ensemble statistics of infinitely large networks. Gavrilenko and Guéguen (1998) have developed a similarly posed renormalization method for fractured media.

It may be possible in principle to use standard renormalization to develop an upscaled description of the hydraulic parameters that pertain to multiphase flow. However, there may be problems in making this application, and this technique has been applied to very few studies of multiphase flows (King et al. 1993). Standard renormalization techniques have been applied successfully to unsaturated zone flow problems (where water forms one phase and air forms the second phase), and some examples are provided by Rockhold (1999) and Rockhold et al. (1999).

\subsection{Volume Averaging and Homogenization Techniques}

By far the most extensive literature regarding the upscaling of saturated and multiphase flow parameters involves two methods known as volume averaging and homogenization. Both spatial homogenization and the method of volume averaging function on the basis of disparate length scales, and both methods produce essentially the same results. The similarity is often ignored by proponents of one method or the other, and that led Bourgeat et al. (1988) to identify the similarities between the two techniques. If any general observations can be made about these two approaches, it is possibly best to say only that homogenization theory tends to appeal more to those with a background in applied mathematics, whereas the method of volume averaging is used frequently by those with an applied science or engineering background.

The method of spatial homogenization was established as a powerful tool for analyzing multiphase systems by the publication of monographs by Bensoussan et al. (1978) and SanchezPalencia (1980), and more recently by Jikov et al. (1994). In this method, one conducts a perturbation expansion in terms of the ratio of two disparate length scales. The method has been applied successfully to a wide range of problems such as diffusion and reaction in porous media (Chang 1982, 1983), flow in deformable porous media (Auriault 1987; Auriault and Boutin 1992), single- and two-phase transport in a double-porosity model of a heterogeneous porous medium (Amaziane 1993; Amaziane and Bourgeat 1988; Arbogast 1993; Bourgeat 1984; Douglas and Arbogast 1990), and many others.

In volume averaging approaches, one seeks to describe the behavior of a system averaged over a particular volume of a porous medium (Whitaker 1999). The approach can be applied in two distinct modes. In the first mode, the method is used to develop equivalent parameters (Ahmadi and Quintard 1996) to upscale structures defined at a fine mesh for use on a coarser mesh. In the second mode, the method is used to develop the effective parameters associated with a representative volume. In this sense, a representative volume can be loosely interpreted as a condition of ergodicity defined earlier; that is to say, a representative volume is defined when an effective parameter for a single realization is the same as the effective parameter averaged over the ensemble of all such volumes. The concept of a representative volume has been recast 
in the framework of representative measurements or representative instrument response functions (Baveye and Sposito 1984; Cushman 1984; Quintard and Whitaker 1994a, 1994b, 1994c.). This extension proposed that properties of porous media should be more generally interpreted in the context of distribution theory, where the distributions employed can be given the physical interpretation of being an instrument response function. In both modes it is necessary to apply boundary conditions on the averaging volume (as it is in homogenization theory), and these boundary conditions are usually chosen to be periodic (for more information about the robustness of this assumption, see the discussion by Pickup et al. (1994).

Two-phase flow and solute transport have been studied extensively from the perspective of volume averaging. A recent review article by Quintard and Whitaker (1999) summarizes much of the work that has been done on the two-phase flow problem in homogeneous and heterogeneous media. Some of the early applications to this problem include the works of Hassanizadeh and Gray (1979a, 1979b), Marle (1982), Cushman (1983), Baveye and Sposito (1984), and Gray and Hassanizadeh (1991a, 1991b). These studies developed the macroscopic conservation equations for mass, momentum, and energy through the use (and development) of formal volume averaging theory (other averages are also defined in some of these works). The goal of these efforts was to identify the macroscopic equations that pertained to transport in porous media and to define the effective parameters in terms of those equations. The explicit representation of the effective parameters in terms of the heterogeneous structure of the porous media and in terms of a closure problem was not emphasized. However, closure problems are the means by which statistical quantities can be incorporated into the prediction of effective parameters such as permeabilities and dispersion coefficients. The effective parameters defined in terms of these statistical quantities provide an explicit connection between the heterogeneous structure in a representative region and the effective parameters. Although these initial studies were important for understanding many issues associated with the complexities of multiphase flows, they were focused primarily on developing the relevant conservation and constitutive equations for homogeneous porous media.

Efforts to relate the heterogeneous structure of the porous media to the definition of the effective parameters (via volume averaging) for two-phase flows were initiated after the original studies using volume averaging. Initial efforts in this area are represented by a sequence of papers by Whitaker $(1986,1994)$, Quintard and Whitaker $(1988,1990 \mathrm{a}, 1990 \mathrm{~b})$, and Lasseux et al. (1996). The study by Whitaker (1986) was the first to develop both the Darcy-scale volumeaveraged continuity and momentum equations (yielding Darcy's law) and the associated closure problem for two-phase flows. The closure problem was subsequently explored in more detail (Whitaker 1994), and a useful transformation of this closure problem was given by Lasseux et al. (1996).

The problem of developing upscaled descriptions for two-phase flows in macroscopically heterogeneous media was explored by Quintard and Whitaker (1988). In that work, a oneequation model is developed under the conditions of quasi-static flow and large-scale mechanical equilibrium. Quasi-static flows were defined as flows where the following conditions were satisfied: 1) the local capillary pressure everywhere in the representative region could be set equal to the macroscopic capillary pressure evaluated at the centroid of the region, and 2) the macroscopic capillary pressure could be set equal to the difference in the macroscopic 
pressures in the two immiscible phases. Mechanical equilibrium means that the Darcy-scale fluid pressure is determined by the pressure-saturation relationships rather than an evolutionary equation. These conditions were relaxed somewhat in an extension of this analysis conducted by Quintard and Whitaker (1990a); however, the effects of large spatial and temporal gradients were not resolved by this work. Numerical experiments reported by Quintard and Whitaker (1990b) have shown that existing one-equation models for two-phase flow in dual-media systems are appropriate under some limiting conditions. However, their results indicate that a large number of cases encountered in practice cannot be satisfactorily represented by a one-equation model.

An upscaling method has been introduced by Durlofsky $(1991,1992)$ and Durlofsky et al. $(1994,1997)$ for multiphase flow that is technically neither volume averaging nor homogenization but does rely on the form of the macroscopic equations that can be derived from these approaches. In this approach, the macroscopic form of the combined mass and momentum conservation equations is posed; then one seeks to determine the macroscopic hydraulic conductivity for a specified region at the coarse grid scale. This is accomplished by 1) assuming that periodic boundary conditions can be imposed on the boundaries of the coarse-grid region, 2) solving for the pressure and velocity fields at the microscopic grid resolution, 3) averaging the velocity field to determine the macroscopic velocity, and 4) calculating the effective hydraulic conductivity on the basis of known average velocity and average pressure fields. Although this method is heuristic in its formulation, it may be possible to show that it is formally equivalent to the method of volume averaging with closure (although this correspondence has not yet been made). Current implementations of this method do not allow for calculation of the off-diagonal components of the hydraulic conductivity tensor, and this could be a limitation under certain circumstances (e.g., see discussion in Pickup et al. 1994). One significant advantage to the method is that it is readily adopted for use with existing computer codes that can solve for the pressure (and velocity) fields for multiphase flows. Potentially, either equivalent or effective conductivities could be determined by this method. Recently, this method has been extended to include a more formal upscaling of the saturation equation, where it has been determined that the saturation equation will contain a dispersion-like term that is caused by fine-scale variations in the velocity field (Efendiev et al. 2000).

\subsection{Full-Resolution Numerical Modeling}

With advances in computational methods there are now cases where the ability to fully resolve the flow problem in a complex heterogeneous media can obviate the need for upscaling. When the fine-scale structure is known (or assumed to be known) deterministically, this technique is particularly powerful because only a single realization of the parameter fields is required. When the fine-scale structure is defined only statistically, it may be necessary to conduct Monte Carlo simulations to determine some measure of the average flow field. However, when there is sufficient separation between characteristic length scales of the finescale heterogeneous structure and the simulation field dimensions, very few (or even one) realizations may be required to achieve good results.

High-resolution modeling has been used extensively in the past to verify various stochastic theories for saturated flow (e.g., Burr et al. 1994; Graham and McLaughlin 1991; Naff et al. 1998a, 1998b; Tompson and Gelhar 1990; Wood and Kavvas 1999a, 1999b). For unsaturated 
flow, high-resolution modeling has been applied less frequently but has become more prevalent with increasing computational capability (e.g., Harter and Yeh 1996a 1996b; Harter and Zhang 1999; Rockhold et al. 1996; Roth and Hammel 1996). In many ways, the connection between high-resolution modeling and various upscaling methods has become inseparable, either because high-resolution models are required to upscale deterministic information from a fine to a coarse grid (e.g., the various volume averaging methods) or because high-resolution models provide one of the few means for testing stochastic theories when only statistical descriptions are available. 



\subsection{Method Recommendations}

Regardless of the methods used to conduct the upscaling, the end result of the upscaling process for the problem of multiphase flows is 1) an upscaled version of the conservation of mass equations and 2) an upscaled version of the conservation of momentum equations. For the problem of flow of water and air in the vadose zone, the equations for water and air are assumed to be fully decoupled. Thus, to predict the water flux in the vadose zone, macroscopic equations of the form

$$
\begin{aligned}
& \frac{\partial \Theta}{\partial t}=\nabla \cdot(\overline{\mathbf{q}}) \\
& \overline{\mathbf{q}}=\mathbf{K}_{e}\left(\Psi, h, \lambda_{1}, \lambda_{2} \ldots\right) \cdot \nabla(\Psi+z)
\end{aligned}
$$

are used. Here $\Theta$ is the macroscopic water content, $\overline{\mathbf{q}}$ is the macroscopic flux, and $\mathbf{K}_{e}$ is the macroscopic (effective or equivalent) hydraulic conductivity function; $H$ is the mean capillary pressure, and $z$ represents the gravitational component of the total hydraulic head. The function $K_{e}$ depends on both the mean capillary pressure, $\Psi$, and on the deviation from the mean capillary pressure, $h$. The deviation, $h$, is defined as the difference between the point value of the capillary pressure, $\psi$, and the mean value, $\Psi$, as follows:

$$
h=\psi-\Psi
$$

In the general form given in Equation (3), the macroscopic flux still depends on the point value of the capillary pressure through $\mathbf{K}_{e}$. The macroscopic problem, then, is essentially specified once one has a representation for the macroscopic hydraulic permeability function, $\mathbf{K}_{e}$. For the problem of determining the effective hydraulic conductivity, the hope is to reduce the amount of information required by determining how $\mathbf{K}_{e}$ depends on certain field statistics of $h$ rather than actual point values of the capillary pressure. For the problem of determining the equivalent hydraulic permeability, the problem is to determine how the macroscopic value $\mathbf{K}_{e}$ can be determined with full knowledge of the point values of the capillary pressure (and any other associated parameter fields that may depend on space).

All upscaling techniques will result in the loss of information; this is essentially the desired effect of upscaling, and it is what leads to the potential for upscaling to improve the tractability of complex problems. The difficulty is to select an upscaling method that preserves important qualities of the problem under consideration. The particular upscaling method that is chosen must be predicated on the basis of a well-posed question as to what information is desired. If, for instance, spatial distributions of water contents around key features are required, methods that uniformly upscale the hydraulic conductivity to a coarse grid will probably not be satisfactory. Such methods might be satisfactory, however, if the only information that is required is the total fluid flux at a compliance boundary. Additionally, it should be noted that all of the upscaling methods assume a priori that a single upscaled equation (with a single macroscopic hydraulic conductivity function) provides an adequate representation of the physical process. However, this kind of a model may not be valid under general conditions. In most of the methods, specific 
constraints have been developed that indicate the conditions under which the methods can be considered valid. When the constraints are not met, it is an indication that the macroscopic manifestation of the physical process is not well represented by the upscaling method and suggests that a different kind of model may need to be applied (e.g., a multi-region model). The development of more general models for upscaling multiphase flows is an area of continuing research.

Each of the upscaling techniques described in Section 2 provides a method for determining macroscopic expressions of the form given in Equations (2) through (4). In the comments that follow, each of these upscaling methods are compared, and recommendations are given.

\subsection{Stochastic Techniques}

Stochastic techniques have the advantages of being the most familiar to hydrologists, and of being relatively simple to implement (i.e., often analytical expressions are available). For the problem of determining the effective saturated hydraulic conductivity, the stochastic techniques are very well developed (e.g., Dagan 1989) but are generally limited to cases where the variance of the structure is small (the so-called "small perturbation" assumption) and well approximated by a log-normal distribution. The stochastic descriptions for flow under unsaturated conditions is perhaps even more tenuous in that nearly all available approaches require that the local hydraulic conductivity be described by an exponential expression of the form of Equation (1). Because the stochastic techniques are applied over regions that are large enough that boundary information of the region does not affect the upscaled parameters, this method is not suitable for nonuniform upscaling (where it may be desirable to maintain certain fine-scale features). It may, however, be combined with other methods to provide a nonuniform coarsening of the problem. For this reason, it is suggested that stochastic techniques be used under only the following limited circumstances:

- A description of the effects of mild heterogeneity is desired for regions that are far from more highly structured regions.

- The characteristic length scale associated with the heterogeneities is small relative to the characteristic length associated with the simulation region.

- An exponential model is an appropriate form for the hydraulic conductivity function.

\subsection{Renormalization Techniques}

Renormalization field theory techniques essentially represent an extended version of the conventional perturbation techniques used in the stochastic theories described above. Therefore, these techniques are applicable under the same circumstances as the stochastic techniques. They have the added benefit that higher-order corrections may be added to the representations for the effective hydraulic conductivity; whether these higher-order corrections are useful in practice has not been addressed in significant detail. 
The standard renormalization techniques have been widely applied for upscaling saturated flow problems and are generally very easy (and computationally inexpensive) to implement. Very few studies (only one has been identified in this review) have been conducted on applying renormalization to multiphase flows. Although standard renormalization techniques are easy to implement, they are based on methods that can not be considered to be as rigorous as some of the other available techniques; therefore, they must be considered to be semi-heuristic. It has been suggested by one of the originators of the method that renormalization may not be a good method when there is a large amount of anisotropy (King and Williams 1994). Unlike oil reservoirs, where the renormalization method has been applied for upscaling multiphase flows, soils tend to be highly anisotropic. Therefore, it is suggested that standard renormalization be used only for the following limited conditions:

- It is necessary to have a fast and computationally nonintensive upscaling method.

- The parameter fields that are upscaled do not exhibit a high degree of anisotropy.

\subsection{Volume Averaging and Homogenization Techniques}

Volume averaging and homogenization methods have had tremendous success in applications for upscaling multiphase flows. These methods generally have a more solid theoretical foundation than the standard renormalization method, but they also tend to be more computationally costly to implement. The tremendous flexibility of these methods generally outweighs the added computational effort, and their ability to be used under very general conditions is an additional advantage. Volume averaging techniques should be considered under the following conditions:

- It is important to obtain an accurate representation of the upscaled parameters.

- Moderate increases in computational effort (relative to standard renormalization) are not prohibitive.

- The system under consideration exhibits a high degree of anisotropy.

- An explicit connection between the fine-scale structure and the macroscopic behavior is desired.

In particular, the semi-heuristic methods described above are very easy to implement, and can be done immediately with minor changes to existing codes (e.g., the addition of periodic boundary conditions). However, with a small amount of additional effort, more formal methods can be employed that have a more sound theoretical basis. With some additional research effort, the volume averaging methods can be extended to cases where a single hydraulic conductivity value is not readily defined (multi-region models) and to cases that improve on the application of periodic boundary conditions.

\subsection{Full-Resolution Numerical Modeling}

In the spectrum of the various methods considered, this method preserves the most information (essentially all the structure is represented within the boundaries of the numerical 
accuracy of the solution method) and is the most computationally intensive. However, with all of the methods presented, there are compromises. The upscaling methods allow approximate solutions to be determined from fine-scale descriptions with a corresponding reduction in cost for calculation of the final coarse-scale solution. However, when one has deterministic structure that must be upscaled by evaluation of the equivalent hydraulic conductivity function over coarse-scale subdomains of the problem, the upscaling technique really represents a way of breaking a large problem up into smaller pieces. The more demands that are placed on the accuracy of the coarse-scale solution, the more closely the computational effort required will approach that of solving the fine-scale problem directly (this is not necessarily true of methods where the effective permeability is needed, because certain statistical assumptions allow the reduction of the total amount of information needed to obtain an accurate solution). This fact is generally recognized, and it still may be necessary to solve a particular problem by an upscaling method simply because it is not possible to solve the fine-scale problem directly (due to computational limitations), whereas solving many smaller problems sequentially is feasible.

With these caveats aside, it should be noted that massively parallel computations are making the fine-scale solution to the flow and transport problems faced by subsurface hydrologists more routine. At Pacific Northwest National Laboratory, the STOMP code (Subsurface Transport Over Multiple Phases [White and Oostrom 1997]) has been developed for massively parallel computation. It has been coupled with a reactive transport code. In principle, this code does not require much more effort or expertise to use than the conventional serial versions of the code. The direct application of this code to a fine-scale (three-dimensional) representation of a subsurface problem has been proven, and it represents a realistic method for the solution of highly resolved flow and transport problems in the subsurface. Fully resolved numerical simulations could be used under the following circumstances:

- Computational resources are not a limiting factor (e.g., massively parallel computational facilities and codes are available for use).

- The physical system is one for which no appropriate upscaling techniques exist (e.g., high contrasts in the fine-scale permeability make the constraints required by the selected models invalid.)

- Detailed information about the field is required (e.g., if the absolute maximum moisture content or flux rate were desired, an upscaling technique might not be suitable because this information may be lost). 


\subsection{Conclusions}

In any practical approach to complex subsurface problems, the methods that are chosen to obtain a solution are always going to be governed by the specific physics of the problem at hand. In many instances, the most practical solution will be one that incorporates one or more of the methods that have been identified in this study. For practical applications, the most reasonable approach is probably one that uses any of these upscaling techniques (provided that the constraints for their validity are met) for upscaling in regions where the variations of the parameter fields are small. For upscaling in regions where the structure is more complex, only the homogenization and volume averaging approaches are probably suitable. Regardless of the upscaling methods that are used, the approach to the problem should be one that allows for nonuniform grid spacing for the final description of the upscaled parameter fields. This is a function of the numerical simulator that is to be used rather than a function of the upscaling method (all of the upscaling methods described could be used to develop coarse-scale descriptions of parameter fields that result in nonuniform grids).

The demand for incorporating increasingly complex phenomena in our representations of subsurface processes seems to be continually increasing (perhaps driven by more stringent regulatory concerns). The need for high-performance computational tools for solutions to these problems is almost inescapable, even with the simplifications that might be afforded by upscaling. Therefore, the process of upscaling can never be fully decoupled from the advent of more powerful computational techniques.

There are still many problems in upscaling that are areas of active research. In particular, the problems associated with sharp contrasts in field properties is possibly the most difficult problem extant. Currently, no techniques exist for upscaling the flow fields associated with multiphase fluids in such high-contrast media. However, if effort were directed to this area of research, new methods could be developed that would have immediate impact on our ability to better represent complex subsurface flows. 



\subsection{References}

Ahmadi A and M Quintard. 1996. Large-scale properties for two-phase flow in random porous media. J. Hydrol., 183:69-99.

Amaziane B. 1993. Global behavior of compressible three-phase flow in heterogeneous porous media. Transport in Porous Media, 10:43-56.

Amaziane B and A Bourgeat. 1988. Effective behavior of two-phase flow in heterogeneous reservoirs, MF Wheeler, ed. Numerical Simulation in Oil Recovery. IMA volumes in Mathematics and its Applications. Springer-Verlag, New York, pp. 1-22.

Arbogast T. 1993. Immiscible displacement in vertically fractured reservoirs. Transport in Porous Media, 12:73-106.

Auriault J-L. 1987. Nonsaturated deformable porous media: Quasistatics. Transport in Porous Media, 2:45-64.

Auriault J-L and C Boutin. 1992. Deformable porous media with double porosity. Quasistatics I: Coupling Effects. Transport in Porous Media, 7:63-82.

Baveye $P$ and G Sposito. 1984. The operational significance of the continuum hypothesis in the theory of water movement through soils and aquifers. Water Resour. Res., 20:521-530.

Bensoussan A, JL Lions, and G Papanicolaou. 1978. Asymptotic Analysis for Periodic Structures. North-Holland Publishing Company, Amsterdam, The Netherlands.

Bourgeat A. 1984. Homogenized behavior of two-phase flows in naturally fractured reservoirs with uniform fracture distribution. Comp. Meth. Applied Mech. Eng., 47:205-216.

Bourgeat A, M Quintard, and S Whitaker. 1988. Eléments de comparaison entre la méthode d'homogénésation et la méthode prise de moyenne avec fermature. C.R. Acad. Sci. Paris, t.306, Série II: 463-466.

Burr DT, EA Sudicky, and RL Naff. 1994. Nonreactive and reactive solute transport in threedimensional heterogeneous porous media: Mean displacement, plume spreading, and uncertainty. Water Resour. Res., 30:791-815.

Chang H-C. 1982. Multiscale analysis of effective transport in periodic heterogeneous media. Chem. Eng. Commun., 15:83-91.

Chang H-C. 1983. Effective diffusion and conduction in two-phase media: A unified approach. AIChE Journal, 29:846-853.

Christakos G, DT Hristopulos, and CT Miller. 1995. Stochastic diagrammatic analysis of groundwater flow in heterogeneous porous media. Water Resour. Res., 31:1687-1703.

Cushman JH. 1983. Multiphase transport equations, I, General equation for macroscopic statistical, local, space-time homogeneity. Transport Theory and Statistical Physics, 12:35-71.

Cushman JH. 1984. On unifying concepts of scale, instrumentation, and stochastics in the development of multiphase transport theory. Water Resour. Res., 20:1668-1676.

Cushman JHE. 1990. Dynamics of Fluids in Hierarchical Porous Media. Academic Press, San Diego. 
Dagan G. 1989. Flow and Transport in Porous Formations. Springer-Verlag, Berlin, p. 465.

Dagan G and SP Neuman, eds. 1997. Subsurface Flow and Transport: A Stochastic Approach. Cambridge University Press, Cambridge, U.K.

Douglas J and T Arbogast. 1990. Dual porosity models for flow in naturally fractured reservoirs, JH Cushman, ed. Dynamics of Fluids in Hierarchical Porous Media. Academic Press, New York.

Durlofsky LJ. 1991. Numerical calculations of equivalent grid block permeability tensors for heterogeneous porous media. Water Resources Research, 27:699-708.

Durlofsky LJ. 1992. Representation of grid block equivalent permeability. Comparison with naïve methods for determining the equivalent perm.

Durlofsky LJ, RC Jones, and WJ Milliken. 1994. A new method for the scale up of displacement processes in heterogeneous reservoirs. Fourth European Conf. on the Mathematics of Oil Recovery. IKU Petroleum Research, Røros, Norway, pp. 1-19.

Durlofsky LJ, RC Jones, and WJ Milliken. 1997. A nonuniform coarsening approach for the scale-up of displacement processes in heterogeneous porous media. Adv. Water Resour., 20:335-347.

Efendiev Y, LJ Durlofsky, and SH Lee. 2000. Modeling of subgrid effects in coarse-scale simulations of transport in heterogeneous porous media. Water Resour. Res., 36:2031-2041.

Gautier Y and B Naetinger. 1994. Preferential flow paths detection for heterogeneous reservoirs using a new renormalization technique. Fourth European Conf. on the Mathematics of Oil Recovery, IKU Petroleum Research, Røros, Norway, pp. 1-17.

Gavrilenko $P$ and $Y$ Guéguen. 1998. Flow in fractured media: A modified renormalization method. Water Resour. Res., 34:177-191.

Gelhar LW. 1993. Stochastic Subsurface Hydrology. Prentice Hall, Englewood Cliffs, New Jersey.

Graham WD and DB McLaughlin. 1991. A stochastic model of solute transport in groundwater: Application to the Borden, Ontario, tracer test. Water Resour. Res., 27:1345-1359.

Gray WG and SM Hassanizadeh. 1991a. Unsaturated flow theory including interfacial phenomena. Water Resour. Res., 27:1855-1863.

Gray WG and SM Hassanizadeh. 1991b. Paradoxes and realities of unsaturated flow theory. Water Resour. Res., 27:1847-1854.

Harter T and TCJ Yeh. 1996a. Conditional stochastic analysis of solute transport in heterogeneous, variably saturated soils. Water Resour. Res., 32:1585-1595.

Harter T and TCJ Yeh. 1996b. Stochastic analysis of solute transport in heterogeneous, variably saturated soils. Water Resour. Res., 32:1585-1595.

Harter T and D Zhang. 1999. Water flow and solute spreading in heterogeneous soils with spatially variable water content. Water Resour. Res., 35:415-426.

Hassanizadeh M and WG Gray. 1979a. General conservation equations for multi-phase systems, 1, Averaging procedure. Adv. Water Resour., 2:131-144. 
Hassanizadeh M and WG Gray. 1979b. General conservation equations for multi-phase systems, 2, Mass, momenta, energy, and entropy equations. Adv. Water Resour., 2:191-203.

Hristopulos DT and G Christakos. 1997. Variational calculation of the effective fluid permeability of heterogeneous media. Physical Review E, 55:7288-7298.

Hunt AG. 1998. Upscaling in subsurface transport using cluster statistics of percolation. Transport in Porous Media, 30:177-198.

Jensen KH and A Mantoglou. 1992. Application of stochastic unsaturated flow theory, numerical simulations, and comparison to filed observations. Water Resour. Res., 28:269-284.

Jikov VV, SM Kozlov, and OA Oleinik. 1994. Homogenization of Differential Operators and Integral Functionals. Springer-Verlag, New York.

King PR. 1987. The use of theoretic methods for the study of flow in a heterogeneous porous medium. J. Phys. Math. Gen., 20:3935-3947.

King PR. 1989. The use of renormalization for calculating effective permeability. Transport in Porous Media, 4:37-58.

King PR, AH Muggeridge, and WG Price. 1993. Renormalization calculations of immiscible flow. Transport in Porous Media, 12:237-260.

King PR and JK Williams. 1994. Upscaling permeability: Mathematics of renormalization. Fourth European Conf. on the Mathematics of Oil Recovery. IKU Petroleum Research, Røros, Norway, pp. 1-17.

Kitanidis PK. 1990. Effective hydraulic conductivity for gradually varying flow. Water Resources Research, 26:1197-1208.

Lasseux D, M Quintard, and S Whitaker. 1996. Determination of permeability tensors for twophase flow in homogeneous porous media: theory. Transport in Porous Media, 24:107-137.

Mantoglou A. 1992. A theoretical approach for modeling unsaturated flow in spatially variable soils: Effective flow models in finite domains and nonstationarity. Water Resour. Res., 28:251-267.

Mantoglou A and LW Gelhar. 1987a. Stochastic Modeling of Large-Scale Transient Unsaturated Flow Systems. Water Resour. Res., 23:37-46.

Mantoglou A and LW Gelhar. 1987b. Capillary Tension Head Variance, Mean Soil Moisture Content, and Effective Specific Soil Moisture Capacity of Transient Unsaturated Flow in Stratified Soils. Water Resour. Res., 23:47-56.

Mantoglou A and LW Gelhar. 1987c. Effective Hydraulic Conductivities of Transient Unsaturated Flow in Stratified Soils. Water Resour. Res., 23:57-67.

Marle CM. 1982. On macroscopic equations governing multiphase flow with diffusion and chemical reaction in porous media. Int. J. Eng. Sci., 20:643-662.

Naff RL, DF Haley, and EA Sudicky. 1998a. High-resolution Monte Carlo simulation of flow and conservative transport in heterogeneous porous media, 1, Methodology and flow results. Water Resour. Res., 34:663-677. 
Naff RL, DF Haley, and EA Sudicky. 1998b. High-resolution Monte Carlo simulation of flow and conservative transport in heterogeneous porous media, 2, Transport results. Water Resour. Res., 34:679-697.

Pickup GE, PS Ringrose, JL Jensen, and KS Sorbie. 1994. Permeability tensors for sedimentary structures. Math. Geol., 26:227-250.

Pollmann DJ, D McLaughlin, S Luis, L Gelhar, and R Ababou. 1991. Stochastic modeling of large-scale flow in heterogeneous unsaturated soils. Water Resour. Res., 27:1447-1458.

Quintard M and S Whitaker. 1988. Two-phase flow in heterogeneous porous media: The method of large-scale averaging. Transport in Porous Media, 3:357-413.

Quintard M and S Whitaker. 1990a. Two-phase flow in heterogeneous porous media, I, The influence of large spatial and temporal gradients. Transport in Porous Media, 5:341-379.

Quintard M and S Whitaker. 1990b. Two-phase flow in heterogeneous porous media, II, Numerical experiments for flow perpendicular to a stratified system. Transport in Porous Media, 5:429-472.

Quintard M and S Whitaker. 1994a. Transport in ordered and disordered porous media I: The cellular average and the use of weighting functions. Transport in Porous Media, 14:163-177.

Quintard M and S Whitaker. 1994b. Transport in ordered and disordered porous media II: Generalized volume averaging. Transport in Porous Media, 14:179-206.

Quintard M and S Whitaker. 1994c. Transport in ordered and disordered porous media III: Closure and comparison between theory and experiment. Transport in Porous Media, 15:31-49.

Quintard M and S Whitaker. 1999. Fundamentals of Transport Equation Formulation for TwoPhase Flow in Homogeneous and Heterogeneous Porous Media, Chapter 1, MB Parlange and JW Hopmans, eds. Vadose Zone Hydrology: Cutting Across Disciplines. Oxford University Press, New York.

Renard P and G de Marsily. 1997. Calculating equivalent permeability: A review. Advances in Water Resources, 20:253-278.

Rockhold ML, RE Rossi, and RG Hills. 1996. Application of similar media scaling and conditional simulation for modeling water flow and tritium transport at the Las Cruces Trench Site. Water Resour. Res., 32:595-609.

Rockhold ML, CJ Murray, and MJ Fayer. 1999. Conditional Simulation and Upscaling of Soil Hydraulic Properties. Characterization and Measurement of Hydraulic Properties of Unsaturated Porous Media, MTh Van Genuchten, FJ Leij, and L Wu, eds. University of California Press, Riverside, pp. 1391-1401.

Rockhold ML. 1999. Parameterizing Flow and Transport Models for Field-Scale Applications in Heterogeneous, Unsaturated Soils. Assessment of Non-Point Source Pollution in the Vadose Zone, DL Corwin, $\mathrm{K}$ Loague, and TR Ellsworth, eds. American Geophysical Union, Geophysical Monograph 108, pp. 243-260.

Roth K and K Hammel. 1996. Transport of conservative chemical through an unsaturated twodimensional Miller-similar medium with steady state flow. Water Resour. Res., 32:1653-1663. 
Russo D. 1992. Upscaling of Hydraulic Conductivity in Partially Saturated Heterogeneous Porous Formation. Water Resour. Res., 28:397-409.

Sanchez-Palencia E. 1980. Non-homogeneous media and vibration theory. Lecture Notes in Physics, 127. Springer-Verlag, New York.

Tompson AFB and LW Gelhar. 1990. Numerical simulation of solute transport in three dimensional, randomly heterogeneous porous media. Water Resour. Res., 26:2541-2562.

Whitaker S. 1986. Flow in porous media II: The governing equations for immiscible, two-phase flow. Transport in Porous Media, 1:105-125.

Whitaker S. 1994. The closure problem for two-phase flow in homogeneous porous media. Chem. Eng. Sci., 49:765-780.

Whitaker S. 1999. The Method of Volume Averaging. Kluwer, Dordrecht.

White MD and M Oostrom. 1997. STOMP: Subsurface Transport over Multiple Phases User's Guide. PNNL-11218, Pacific Northwest National Laboratory, Richland, Washington.

Wood BD and ML Kavvas. 1999a. Correction to "Stochastic solute transport under unsteady flow conditions: Comparison of theory, Monte Carlo simulations, and field data." Water Resour. Res., 35:2889.

Wood BD and ML Kavvas. 1999b. Stochastic solute transport under unsteady flow conditions: Comparison of theory, Monte Carlo simulations, and field data. Water Resour. Res., 35:20692084.

Yeh T-CJ, LW Gelhar, and AL Gutjahr. 1985a. Stochastic Analysis of Unsaturated Flow in Heterogeneous Soils, 3, Observation and Application. Water Resour. Res., 21:465-471.

Yeh T-CJ, LW Gelhar, and AL Gutjahr. 1985b. Stochastic Analysis of Unsaturated Flow in Heterogeneous Soils, 2, Statistically Anisotropic Media with Variable x. Water Resour. Res., 21:457-464.

Yeh T-CJ, LW Gelhar, and AL Gutjahr. 1985c. Stochastic Analysis of Unsaturated Flow in Heterogeneous Soils, 1, Statistically Isotropic Media. Water Resour. Res., 21:447-456. 


\section{Distribution}

No. of

Copies

\section{Offsite}

2 DOE Office of Scientific and

Technical Information

Mark Rockhold

1110 NW Grant Ave,

Corvallis, OR 97330

\section{Onsite}

3 Fluor Daniel Northwest

EJ Freeman

FM Mann

R Khaleel
No. of

Copies

22 Pacific Northwest National Laboratory

B4-43

$\mathrm{HO}-22$

B4-43
DH Bacon

SQ Bennett

MJ Fayer

CT Kincaid

BP McGrail

PD Meyer (5)

KP Saripalli

CS Simmons

JE Szecsody

AL Ward

BD Wood (3)

K9-33

K9-70

K9-33

K9-33

K6-81

BPO

K6-81

K9-33

K3-61

K9-33

K3-61

Information Release (5)

K6-06

Distr.1 